\section{Workshop on the Development and Function of Reproductive Organs}

\author{
Rome, 14-17 September 2008
}

The XIV Workshop on the Development and Function of Reproductive Organs was held in Rome, from 14-17 September, 2008, at the Congress Centre of the University of Tor Vergata of Villa Mondragone (Rome, Italy). The Workshop was conceived to be a survey of the events from the formation of the gamete precursors, the primordial germ cells, to the development of the preimplantation embryo through female and male gametogenesis in the mouse experimental model. It was divided into six topics including classic and highly-debated current subjects in the field of the biology of reproduction: mammalian primordial germ cells, the formation of the gonads, stem cells and germ cells, gametogenesis from birth to adult (male), gametogenesis from birth to adult (female), and finally fertilization and preimplantation embryo.

\section{Mammalian primordial germ cells}

An overview of mouse primordial germ cell (PGC) development and the renewed interest in PGC biology as the guardian of differentiation totipotency were the subjects of the introduction by Massimo De Felici to the first section on mouse PGCs dedicated to Anne McLaren. This great scientist who died last year has been lovingly remembered as the initiator and mentor of all the present studies on mouse PGCs.

Talking about one of the most loved subjects of Anne McLaren's research, i.e. germ line formation, Kirstie Lawson of the MRC Human Genetic Unit in Edinburgh, opened the section reviewing the studies that she herself begun 25 years ago and that have led today to establishment of the sequential stages of PGC specification in the mouse embryo. A perceptive conjecture by Anne McLaren in 1980 predicted that the selection of cells to form the germline in the mouse depended on their position in the epiblast. Today we know that fascinating mechanisms of gene repression and activation really do occur in PGC precursors in the epiblast. After genome-wide epigenetic reprogramming, PGCs are then specified during early gastrulation in a group of about 40 cells located in the extraembryonic mesoderm, as Lawson with elegant fate mapping experiments had predicted. In 1981, Anne McLaren in the book Germ Cells and Soma wrote "the potential for totipotency resides in all cells" and "to realize this potential for totipo-

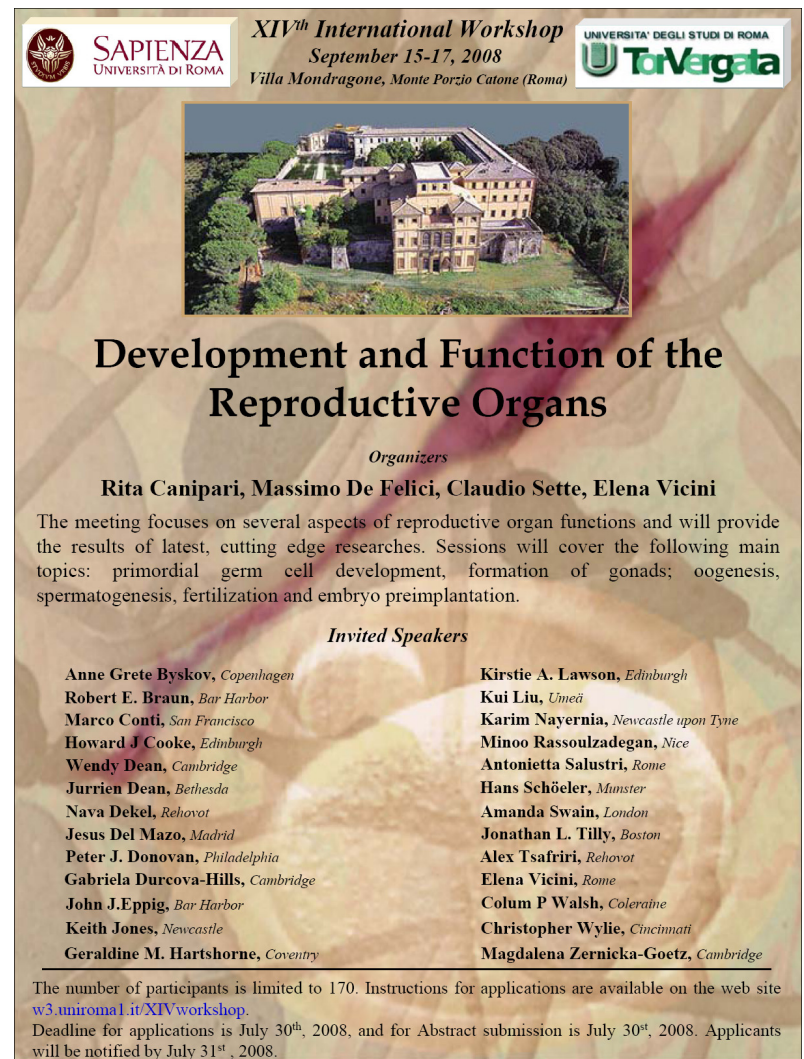

tency, the cell must be exposed to the appropriate environment". In their lectures, Peter Donovan of the University of California, Irvine (USA), and Gabriella Durkova-Hills of the Wellcome Trust Cancer Research UK, Gurdon Institute, University of Cambridge (UK), addressed the topic of the relationships between embryonic stem cells (ESCs) and PGCs. Although PGCs are lineage committed cells and have limited self renewal capability, they are the only cells of the mammalian postimplantation embryo able to form pluripotent and self renewing cell lines both in vivo (Embryonal Carcinoma Cells, ECCs) and"in vitro (Embryonic Germ Cells, EGCs). This unique capability is due to the expression in PGCs of master transcription factors for pluripotency such as OCT4, SOX2 and nanog and also, probably to a peculiar epigenetic state of the genome. While the function of these stem/germ cell genes in PGCs is little understood, it is possible that they use them for some germ-cell specific function, rather than for the regulation of pluripotency. During normal development, PGC pluripotency must be held at bay by repressors of transcription and transduction. At the same time, a strict control of the cell cycle assures the correct progression of their differentiation and a finite number of mitoses. 
In some way, both repression and cell cycle control are impaired when PGCs are induced to transform into ECCs or EGCs. While it was known that in culture the combined action of $\mathrm{KL}$, LIF and FGF-2 is required for PGC-EGC transition, little was known about the molecular mechanisms underlying this phenomenon. The key role of epigenesis and cell cycle control in maintaining the normal PGC phenotype clearly emerged from the Durcova-Hills laboratory. She reported that trichostatin $A$, an inhibitor of histone deacetylase, is a highly potent agent that can replace FGF-2 to induce dedifferentiation of PGCs into EGCs and that a key event in this process is the downregulation of Blimp1, a repressive transcription factor playing a crucial role in the specification and maintenance of the early germ cell lineage. Interestingly, downregulation of Blimp 1 is associated with upregulation of $c-M y c$ and $K / f-4$, which represent two of the four factors (the other two being Oct4 and Sox2, already highly expressed in PGCs) known to promote reprogramming of somatic cells to the pluripotent state.

\section{Formation of the gonads}

The adrenal cortex and gonads are the main steroid-producing tissues in mammals. Although they produce different steroid hormones in the adult, histological and molecular studies have shown that they share a common precursor during development. Expression analysis of the nuclear hormone receptor SF-1 (steroidogenic factor 1), an essential factor for the development of both gonads and adrenals, suggests that these two tissues originate from a so-called adrenogonadal primordium (AGP) present in the embryonic day 9 (E9.0) mouse. Amanda Swain of the Institute of Cancer research in London (UK), showed a direct correlation between levels of $S f-1$ expression in the AGP and induction of adrenal and gonad development. With doses of Sf-1 expression in the AGP at $36 \%$ of wild-type levels, as seen in Cited $2^{/-}$embryos, adrenal development does not occur, but gonad development is initiated, although differentiation is impaired. Increasing the dose of Sf-1expression, as seen in Cited2 ${ }^{\prime}$ - $W t^{1+-}$ mice, is enough to ensure adrenal specification, although the organ is smaller. Cited2 acts as a cofactor for the transcription factor Wt1 to regulate the levels of SF-1. Adrenal cortex development is more sensitive to SF-1 dosage than gonadal development. In this regard, it is to be pointed out that a little later, in the testis, SF-1 and SRY cooperatively upregulate Sox9expression and then, together with SF-1, SOX9 also binds to the enhancer to help maintain its own expression after that of SRY has ceased and trigger Sertoli cell differentiation.

Although mammalian sex is determined genetically, the sex specific development of germ cells as sperm or oocytes is initiated by cues provided by the gonadal environment. During embryogenesis, PGCs in the ovary enter meiosis, thereby committing to oogenesis. In contrast, germ cells in a testicular environment do not enter meiosis until puberty. The mechanism by which meiosis is triggered in the ovary has been a subject of considerable debate. The point at issue has been whether, in the developing ovary, initial entry into meiosis occurs spontaneously and cell-autonomously, perhaps regulated by a molecular clock or whether this step is induced by surrounding somatic tissue. Recent findings indicate that exposure to retinoic acid (RA) controls whether mouse PGCs enter meiosis or not. Ian Adams and Gabriel Livera in their short talks presented interesting data supporting the existence of an unidentified compound secreted by fetal Sertoli cells with meiotic preventing action independent of the RA/Cyp26b1 system. Thus, the role of RA in meiosis seems to have been overrated.

Entering into meiosis is accompanied and followed by a series of complicated processes involving DNA double strand breaks, the formation of the synaptonemal complex (SC), chromosome pairing and synapsis, DNA repair and recombination, the end of which is the exchanging of genetic information during chromosome crossing over in prophase I. The study of meiosis in mammals has been performed at a variety of levels ranging from the biochemical to the cytogenetic, but it has not been easily amenable to the genetic approaches available in model organisms such as Saccharomyces cerevisiae and C. elegans. These genetic approaches have been productive in revealing an increasing number of proteins known to be involved in meiosis. However, lack of sequence conservation has made extrapolation to mammals difficult. Using a different approach, expression profiling was used to find new components of the meiotic machinery. Components of the mammalian synaptonemal complex (SC) have been identified only in the last 15 years, and mouse genetic approaches have started revealing the importance of this structure only in the past 5 years. Howard Cooke of the MRC Human Genetic Unit in Edinburgh (UK), reported a model for SC formation based on such experimental approaches and mutation of the four known protein components of the SC central element (Syce1 and 2, Tex12 and Sycp1).

The vast majority of oocytes which are formed in the fetal ovary and progress through prophase I do not survive beyond birth. Possible reasons for their loss include the elimination of nonviable genetic constitutions arising through meiosis and the scarcity of pro-survival factors. Moreover, the precise relationship between meiotic stages and prenatal apoptosis of oocytes remains elusive. The results reported by Geraldine Hartshorne of the University of Warwick, Coventry (UK), showed that oogenesis in the prenatal ovary both in mouse and human is indeed influenced by a number of parallel processes contributing to the survival or death of oocytes. Thanks also to the in vitro culture model, the emerging view is that fetal oocytes possess and are able to activate several pathways of various forms of cell death, probably as a consequence of different death stimuli. However, casual correlation among such different cell death pathways remains to be determined and stimuli causing their activation clarified.

\section{Stem cells and germ cells}

Over the last few years, scientists working in the field of the biology of reproduction have been shocked by reports of the possibility of generating male germ cells and oocyte-like cells in culture from a variety of somatic cells including ESCs and ECCs, skin, bone marrow and pancreatic stem cells as well. Although no one doubted that germ cells could be derived from ESCs in vivo and early PGCs from cultured epiblast, it appeared unbelievable to people aware of the sophisticated mechanisms regulating the complex interactions between germ cells and their somatic environment during gametogenesis, that germ cells in advanced stages of differentiation could be obtained simply in a dish from so disparate types of somatic cells. Two of the most eminent authors 

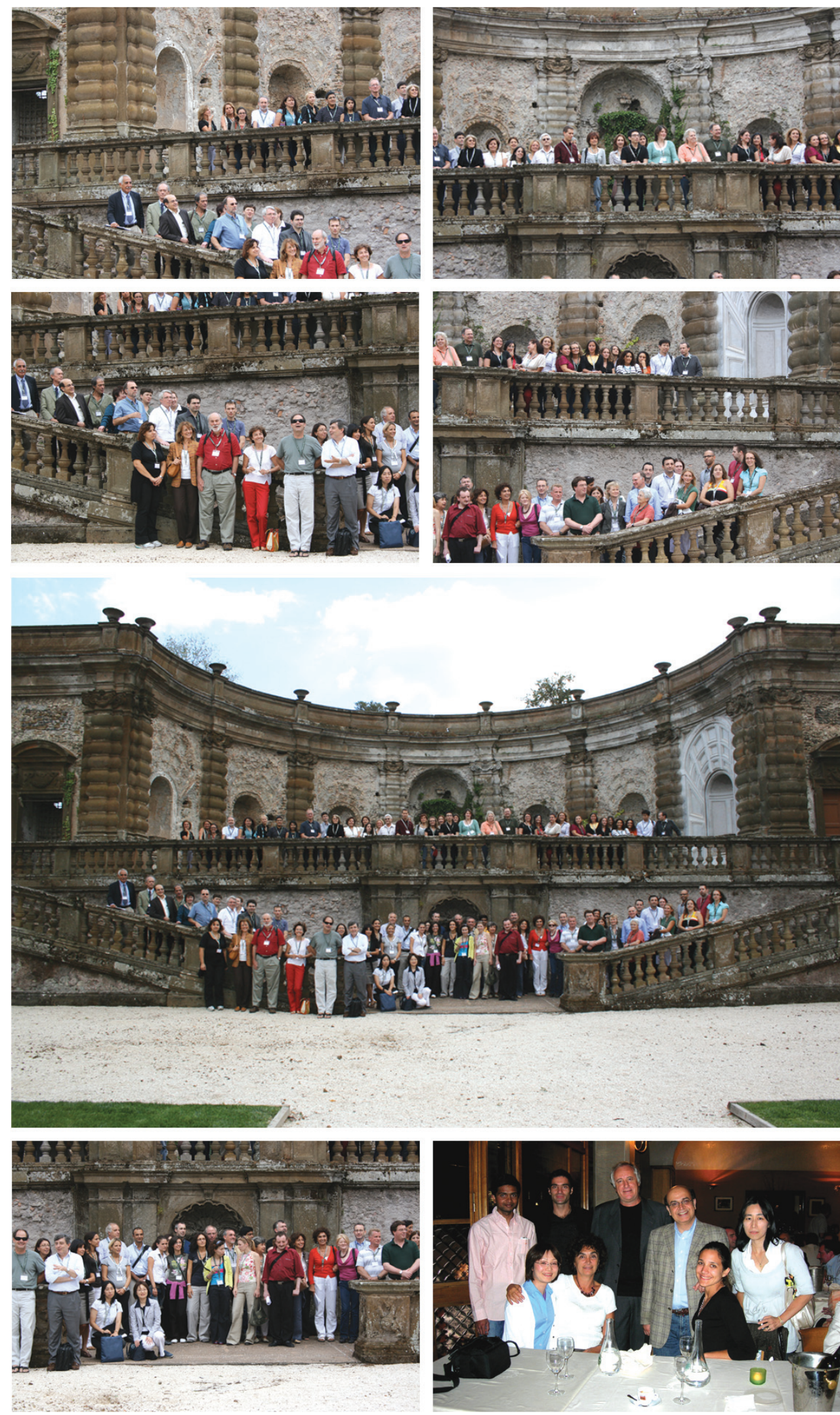

Group photographs of the participants in the XIV Workshop on the Development and Function of Reproductive Organs (Rome, 14-17 September 2008). of this upheaval, Hans Schöler and Karin Nayernia, opened this third session. Nayernia, professor of stem cell biology at Newcastle University (UK), reviewed the research and strategies leading his group to produce apparently haploid male germ cells from ESCs and bone marrow stem cells and highlighted the largely shared necessity for a better morphological and molecular characterization of the early and late stages of this extraordinary process occurring in culture. Prof. Schöler, now director of the Max Planck Institute for Molecular Biomedicine in Münster (Germany), talked about the, in some way, inverse process to produce pluripotent stem cells from adult spermatogonia stem cells. Researchers have long suspected that spermatogonial stem cells (SSCs), which males need for continuous sperm production, might have further potential. But only in 2004 did scientists finally succeed in growing SSCs in culture from mice. Using SSCs obtained from the testes of neonatal or adult mice, researchers have recently been able to redirect the cell development in culture to form so-called «multipotent germline stem cells" (mGSCs) or "multipotent adult germline stem cells" (maGSCs) or "multi-potent adult spermatogonial-derived stem cells" (MASCs). Robust and reproducible methods for deriving adult pluripotent stem cells would have an immense impact on regenerative medicine. Such cells would circumvent the ethical and technical problems associated with embryonic stem (ES) cells, and the possibility of autologous transplantation would avoid the risk of immunorejection. However, reprogramming of mouse and human somatic cells into pluripotent stem cells, known as induced pluripotent stem (iPS) cells, reported for fibroblasts, requires the introduction of the viral expressed transcription factor quartet Oct4, Sox2, cMyc and KIf4. Schöler showed that pluripotent stem cells termed germline pluripotent stem (gPS) cells can be obtained from mouse self renewing and unipotent SSCs which already express Oct4 and K/4 by non-viral induced expression of Sox2and $c-M y c$, even after clonal expansion of single SSCs.

Elena Vicini of the Department of Histology and Medical Embryology, of 
the University of Rome La Sapienza (Italy), presented new convincing evidence about the heterogeneity of SSCs based on side-population and dye-based cell staining analyses. Ex vivo immuno-phenotyping and intra testicular tubular transplantation further provided compelling evidence for SSC heterogeneity. Eventually, she showed for the first time evidence for a previously hypothesized asymmetric SSC division as a possible source of such heterogeneity.

The session ended with a lecture by Jonathan Tilly of the Vincent Centre for Reproduction, Massachusetts General Hospital/Harvard Medical School, Boston (USA). In the field of reproductive biology, there has been a longstanding dogma that mammalian females produce a finite number of oocytes in the prenatal phase of life and this production ceases after birth given the absence of germ stem cells in the female. Over the past four years, Tilly and his collaborators produced data which contradict this dogma and support the postnatal development of oocytes and follicles in female mouse ovaries. The mechanism of de novo oocyte formation has been proposed to involve a contribution from the bone marrow to the germ cell pool, via the bloodstream, but little has been reported about the nature of this elusive bone marrow population with germ cell potential and/or its ability to stimulate new oogenesis in the adult ovary. Given the harsh debate between Tilly and critics of his group's results, there was much impatient waiting for new evidence against the established dogma. Tilly said that they have now developed a transgenic mouse expressing enhanced green fluorescent protein (EGFP) under the promoter of the stimulated by retinoic acid 8 (Stra8) gene, known to be specifically expressed in premeiotic germ cells; this allows the identification of "oogenic" cells in the ovary. By expanding this cell population in vivo with trichostatin $A$, they are now in the phase of isolating hundreds of such Stra8 expressing cells from the adult ovary. In addition, Tilly said that they have also generated three novel gene "knock-in" mouse lines that will provide a means to ablate these cells in a lineage-and temporal specific manner.

\section{Gametogenesis from birth to adult (male)}

This session touched the classical topic of spermatogenesis from three different perspectives: the effects of endocrine disruptors and of ablation of androgen receptors on testis development and spermatogenesis. The unusual third perspective involved a curious epigenetic gene modification termed paramutation.

Jesus del Mazo of the CSIC, Madrid (Spain) summarized a huge series of data which his group has accumulated over the years during the course of two EU projects aimed to investigate, using a variety of gene expression analyses, the risk for spermatogenesis of exposure both in vivo and in vitro to xenobiotic compounds during critical periods of testis development. These studies allowed the elaboration of transcriptional profiles associated with the deregulation of genes involved in diverse pathways (including the respiratory chain, oxidative stress, ribosomal proteins, cell-cell interaction, apoptosis and cell metabolism), the establishment of transcriptome patterns for specific compounds and the definition of potential transgenerational transmitted effects.

Proper functioning of the mammalian testis is dependent upon an array of hormonal messengers acting through endocrine, paracrine and autocrine pathways. Within the testis, the primary messengers are the gonadotropins, follicle stimulating hormone and luteinizing hormone, and the androgens. Abundant evidence indicates that the role of gonadotropins is to maintain proper functioning of testicular somatic cells. Androgens are known to act in the testis by binding to receptors on Sertoli cells and Leydig cells, but it remains unclear if androgen receptors are needed or even present on germ cells. "Do androgens provide instructive signaling to germ cells?" was the title of the lecture given by Robert Braun of the Jackson Laboratory, Bar Harbor, Maine and Washington State University, Pullman, WA (USA). To identify potential targets of androgen signaling in the testis, Braun and collaborators have analyzed the transcriptional profile of adult testes from mice hypomorph for the androgen receptor $(\mathrm{Ar})$ alone or in combination with Sertoli cell-specific Ar ablation. Using Affymetrix mouse genome arrays (22,000 transcripts), they found 62 transcripts in the Armutants whose expression level differed by greater than 2 -fold compared with wild type. They also found that more transcripts were up-regulated than down-regulated. Twelve transcripts were uniquely affected, and 16 transcripts were more severely affected in Sertoli cell-specific Ar ablation compared with hypomorphic $A r$ mutants. Finally, they found at least one conserved ARE (androgen response element) in $65 \%$ of the misregulated genes. The transcripts affected by these $\mathrm{Ar}$ mutations encode a diverse array of proteins whose molecular functions support the contention that AR supports spermatogenesis in both a permissive and instructive fashion.

The term "paramutation" describes a genetic behavior in which the regulatory state of specific alleles is heritably altered through interactions with their homologous partners in trans. This behavior constitutes an exception to the Mendelian principle that alleles segregate from a heterozygous state unchanged. Paramutations have been best characterized at loci encoding transcriptional regulators of pigment biosynthesis in maize, but similar behaviors have been described in other plant and animal systems, most recently in mice. Minoo Rassoulzadegan of INSERM of the University of Nice-Sophia Antipolis, Nice (France), reported a paramutation of the mouse Kit gene, crucial for spermatogenesis, in the progeny of heterozygotes with the null mutant Kit (tm1Alf) (a lacZ insertion). In spite of a homozygous wild-type genotype, their offspring maintain, to a variable extent, the white spots characteristic of Kit mutant animals. Efficiently inherited from either male or female parents, the modified phenotype results from a decrease in Kit messenger RNA levels with the accumulation of non-polyadenylated RNA molecules of abnormal sizes. Sustained transcriptional activity at the postmeiotic stages, at which time the gene is normally silent, leads to the accumulation of RNA in spermatozoa. Microinjection into fertilized eggs of either total RNA from Kit (tm1Alf/+) heterozygotes or of Kitspecific microRNAs, induced a heritable white tail phenotype. Finally, Rassoulzadegan reported that her group has recently identified another paramutation in a mouse model of hypertrophic cardiomyopathy (HCM).

\section{Gametogenesis from birth to adult (female)}

When a woman is born, her ovaries already contain a full supply of oocyte-containing follicles which she will need in her reproduction lifetime. Groups of these follicles are recruited and 
the oocytes begin growing at about 13 years of age and are gradually released to be fertilized, usually at the rate of one per month, until she is about 50 years old. The duration of fertility of a female is determined by the initial size of her follicle pool and by the rate of its activation and depletion. While growth factors, such as Kit Ligand (KL), have been identified as inducers of early oocyte growth, the intraoocyte signaling pathways which control follicle recruitment and oocyte growth are largely unknown. In his elegant talk, Kui Liu of the Department of Medical Biochemistry and Biophysics, of the Umea University, Umea (Sweden), presented compelling data suggesting that the phosphatidylinositol 3-kinase (PI3K) pathway in oocytes may play a crucial role in regulating oocyte growth and the activation of primordial follicles. In fact, the deletion in mouse oocytes of the Ptengene, a major negative regulator of $\mathrm{PI} 3 \mathrm{~K}$, caused infertility in early female adulthood. In the absence of Pten, the researchers found that the oocyte-containing follicles of mice were activated rapidly and oocytes begin to grow at an early age, thus causing depletion of the animal's oocytes much sooner than is normal, a situation similar to that of premature ovarian failure (POF) in humans. Interestingly, PI3K and PTEN are major antagonizing players of the intracellular pathways activated by the binding of $K L$ to its receptor KIT.

In mammals, some genes categorized as imprinted genes are exclusively expressed either from the maternal or paternal allele. This parental-origin-specific gene expression is regulated by epigenetic modification of DNA methylation in differentially methylated regions (DMR), which is independently imposed during oogenesis and spermatogenesis by de novo methyltransferases (DNMTs). It is known that methylation of DMRs in the female germ line is established during oocyte growth phase mainly by DNMT3A and DNMT3L. However, the cause of the progression of methylation of DMRs, due to either aging of mice or oocyte growth-size was unclear up to now. To clarify this issue, Column P. Walsh of the Stem Cells and Epigenetic Research Group, Centre for Molecular Biosciences, School of Biomedical Sciences, University of Ulster, Northern Ireland (UK), in collaboration with Massimo De Felici (University of Rome Tor Vergata, Italy), using a suitable in vitroculture system for growing mouse oocytes studied whether oocyte growth induction by $\mathrm{KL}$ was associated with the methylation of DMRs of some imprinted genes. Their results indicate that the signals triggering oocyte growth and DNA methyltransferase expression are separate from those required for imprinting, with the former being triggered solely by oocyte growth and the latter likely requiring progression to the antral follicle stage. These results underline the interplay between oocytes and their supporting follicular cells and indicate that de novo methylation does not just rely on an oocyte-intrinsic program, but rather is dependent on outside signals produced by, or mediated through, the surrounding granulosa and thecal cells.

Although granulosa cells provide essential nutrients and stimuli for oocyte growth and development, oocytes are not merely passive recipients of such support, but rather active regulators of follicular development. It is now clear that communication between oocyte and follicular cells is bidirectional. What is quite surprising is that the oocyte can even instruct follicular cells to produce compounds necessary for its metabolism which it is unable to synthesize. John J. Eppig of The Jackson Laboratory, Bar Harbor (USA) and his group has largely contributed to the exploration of bidirectional communication between oocytes and companion follicular cells. It has been known for many years that oocytes only poorly transport some amino acids or metabolize glucose as an energy source. Oocytes are also highly deficient in their ability to carry out de novo cholesterol synthesis from acetate. Data presented at this Meeting demonstrate how oocytes, to compensate for these deficiencies, stimulate cumulus cells to increase the steady sate levels of mRNAs encoding proteins involved in L-alanine uptake, glycolysis and de novo cholesterol synthesis.

The mammalian oocyte is surrounded by several layers of follicle cells termed cumulus granulosa cells that nurture the oocyte during its development and actively participate in the process of ovulation. After the ovulatory LH surge, a distinctive program of extracellular matrix production is initiated in the cumulus-oocyte complex. This process, known as cumulus expansion or mucification, involves synthesis of a backbone of long hyaluronan oligosaccharide chains that are cross-linked by a complex of hyaluronan binding cell surface and extracellular matrix proteins and proteoglycans. Active components of the cumulus matrix are synthesized directly by cumulus cells under the control of endocrine- and oocyte-derived factors, secreted by mural granulosa cells, or enter the follicle in blood plasma. Appropriate composition and assembly of the cumulus matrix is essential for ovulation, efficient passage of the oocyte through the oviduct, and for fertilization.

Antonietta Salustri, from the Department of Public Health and Cell Biology, University of Rome Tor Vergata (Italy), presented new findings on the PTX3/I-alpha-I/TSG6 interaction which might provide new insights into the mechanisms by which the viscoelastic matrix of the cumulus is assembled.

Studies on $P t \times 3^{/-}$mice suggested that oocytes ovulated by $P T X 3^{-/-}$mice can be fertilized in vitro, indicating that defective cumulus expansion is the sole cause for in vivofertilization failure and that PTX3 acts as a central "node" in cumulus matrix organization by establishing multivalent contacts to the HAbinding protein tumor necrosis factor-stimulated gene- 6 protein (TSG-6) and the serum protein inter- $\alpha$-inhibitor. These findings strongly suggest that the cumulus cell matrix plays a crucial role for in vivosperm recruitment and guidance towards the oocyte, a condition likely circumvented under in vitro fertilization settings, due to the high concentration of sperm.

At the time of ovulation, the LH surge induces oocyte meiotic maturation and enables the oocyte for fertilization and developmental competence. In recent years, Marco Conti from the Center for Reproductive Sciences, University of California at San Francisco, San Francisco CA (USA) has demonstrated that members of the epidermal growth factor (EGF)-like growth factor family are likely mediators of $\mathrm{LH}$ action in the follicle. Specifically, amphiregulin (AREG), epiregulin (EREG) and betacellulin (BTC) are rapidly induced by LH or its analog, human chorionic gonadotropin (hCG) and are thought to function in an autocrine and paracrine manner to propagate LH signals throughout the preovulatory follicle. In order to define whether EGF-like growth factor production affects oocyte developmental competence, oocytes from amphiregulin null $\left(\right.$ Areg $\left.^{-)}\right)$mice were used for in vitro fertilization and preimplantation embryo development. Denuded oocytes or cumulus/oocyte complexes derived from these mice were fertilized at a reduced rate compared to wild type controls. 
Moreover, the efficiency of in vitro embryo development to the blastocyst stage was significantly reduced in the Areg $^{\prime-}$ embryos. Counting the number of cells in morulae indicated that the rate of cell division is reduced in the Areg ${ }^{-/}$embryos. This phenotype could be partially rescued by exposure of the cumulus oocyte complex to recombinant AREG. Areg is not produced by the oocyte, but its mRNA is present in cumulus cells.

A controversial subject in oocyte meiotic resumption is the maintenance of cAMP levels necessary for the prevention of meiotic maturation in germinal vesicle (GV) oocytes which are competent to re-enter the cell cycle. For many years, a common tenet has been that oocytes are not able to produce sufficient cAMP to maintain the meiotic blockade and that the somatic compartment provides the oocyte with a pool of cAMP through the extensive network of transzonal projections and gap junctions connecting the oocyte to cumulus cells. More recent studies have revisited the idea of oocyte autonomous production of CAMP and its role in maintaining meiotic arrest. Nava Dekel from the Department of Biological Regulation, Weizmann Institute of Science, Rehovotn (Israel) presented data showing that, in addition to the termination of gap junction communication, LH upregulates the activity of PDE3A, thus enhancing the rate of CAMP degradation and further facilitating the reinitiation of meiosis.

Moreover, this researcher observed an immediate, transient elevation of intraoocyte cAMP concentration in follicle-enclosed oocytes (FEOs) exposed to $\mathrm{LH}$. As expected, this elevation of cAMP within the oocytes was followed by activation of PDE3A above basal levels. Both the immediate $\mathrm{LH}$-induced elevation of intraoocyte CAMP and subsequent PDE3A activation could not be demonstrated in FEOs which were exposed to the gap junction blocker, CBX. They conclude that oocyte-specific PDE3A indeed mediates $\mathrm{LH}$ action in the resumption of meiosis. $\mathrm{LH}$-stimulated increase in the follicular concentration of cAMP is followed by a gap-junction mediated transient elevation of this nucleotide in the oocyte, taking place just prior to the termination of cell-to-cell communication in the ovarian follicle. Exposure of oocyte PDE3A to increasing cAMP levels upregulates its hydrolyzing activity. This response brings about a rapid and effective decrease in the levels of CAMP in the oocyte, thereby facilitating the resumption of meiosis.

The session ended with Alex Tsafriri from the Department of Biological Regulation, Weizmann Institute of Science, Rehovot (Israel). While the essential role of steroids in the mediation of gonadotropic stimulation of oocyte maturation in amphibians and fish is well established, much more controversial is the notion that they can mediate $\mathrm{LH}$ action on resumption of meiosis in mammals. In his presentation, Tsafriri presented some evidence that led him to conclude that steroids are not necessary for the resumption of mammalian meiosis. Nevertheless, steroids are probably involved in follicular growth, somatic-cell differentiation and the acquisition of developmental competence of mature oocytes.

\section{Fertilization and the preimplantation embryo}

Oocyte quality is a major factor governing a woman's fertility and embryo development. Quality is poor when chromosome segregation errors occur during the first meiotic division. Such errors, which include trisomy 21 (Down's Syndrome), affect between $20-40 \%$ of all human oocytes and go on to produce mostly non-viable, aneuploid embryos, thus making chromosome segregation errors the leading cause of early embryo loss. Keith Jones from Human Physiology, School of Biomedical Sciences, University of Newcastle, Callaghan (Australia), presented data showing that oocyte susceptibility to chromosome mis-segregation is due to its peculiar and unique regulation of the Spindle Assembly Checkpoint (SAC) target: the Anaphase-Promoting Complex (APC) and its two co-activator proteins CDC20 and $\mathrm{CDH1}$. The oocyte uses a stalling mechanism to delay meiotic division based on APC-CDH1 activity, until chromosomes can be segregated equally. This mechanism is not observed in adult cells and is inherently error prone. He postulated that this activity provides a ready target for mis-regulation, and could account for the increasing rates of aneuploidy as women age.

The interesting issue of cell-fate determination in early embryogenesis was raised by the presentations of Magdalena Zernicka-Goetz from The Gurdon Institute, University of Cambridge, (UK) and Jurrien Dean from the Laboratory of Cellular and Developmental Biology, NIDDK, National Institutes of Health, Bethesda (USA). Following fertilization, the mouse embryo undergoes three mitotic cell divisions before compacting at eight cells to form individually polarized cells. The first cell fate decision in early mouse development is whether to become part of the pluripotent inner cell mass (ICM) or the extra-embryonic tissue, the trophectoderm. The genesis of these two lineages appears to occur in two stages. First, cells are allocated to different inside and outside positions via asymmetric divisions. Outer cells may divide asymmetrically, to generate one outer and one inner daughter cell, or symmetrically, in which case both daughters remain outside. There are two waves of these a/symmetric divisions: one at the eight- to 16-cell stage transition and the other at the 16- to 32-cell stage. Then, the cells in these different positions become specified; they become committed to restricted developmental fates. This involves interactions between different transcription factors which specify trophectoderm and ICM cell fate. Two hypotheses have been put forward to explain how inside and outside cells might start to differ from each other. The first stresses the importance of cell position (the «inside-outside» hypothesis). Thus, when the positions of cells are changed, they develop according to their new position. The second hypothesis stems from the discovery that cells become polarized along the apicalbasal axis at the eight-cell stage. When the polarized cells divide, outside cells inherit different cellular components than inside cells. Magdalena Zernicka-Goetz found that these hypotheses are not mutually exclusive and showed how both cell position and polarization together regulate the expression of cell fate-determining genes. The commitment of outside cells to trophectoderm is mediated at the blastocyst stage by $C d \times 2$. However, $C d \times 2$ can also act earlier to influence cell polarization and the pattern of symmetric versus asymmetric divisions that allocate cells to either trophectoderm or ICM. This early expression of Cdx2 commences at the 8-cell stage and is heterogeneous between blastomeres. Naturally occurring, or experimentally induced, high levels of $C d \times 2$ lead to a higher frequency of symmetric divisions and, consequently, allocation of more cells to trophectoderm. Conversely, reduced $C d \times 2$ expression leads to an increase in asymmetric divisions and contribution to the ICM. The level of $C d x 2$ expression affects the extent of cell polarization, but cell 
polarity in turn leads $C d \times 2$ transcripts to become asymmetrically distributed in blastomeres. Thus, the asymmetric divisions that generate inside and outside cells are truly asymmetric in terms of differentially distributing fate instructions between daughters. Such a feedback loop between cell polarization and gene expression ensures that the switch from pluripotency to differentiation is robust. Finally the heterogeneity of $\mathrm{Cdx} 2$ expression between blastomeres does not arise at random, but depends on cell history and specifically upon how the contents of the zygote become partitioned between the cells. Underlying this are differential levels of specific epigenetic modifications between 4-cell blastomeres instrumental in assigning different developmental potential to these cells; those with higher levels of methylated arginines in histone $\mathrm{H} 3$ are most pluripotent. Together, these results indicate that the history of cells affects their later interactive capacity. Such bias in development would maximize chances of developmental success while retaining developmental plasticity.

Jurrien Dean (NIDDK, National Institutes of Health, USA) presented data showing that during oocyte growth and maturation, mammalian oocytes accumulate proteins required for successful fertilization and early embryogenesis. Mater, Floped, Tle6 and Filia are oocyte-specific genes encoding proteins that form a subcortical maternal complex (SCMC). Although individual transcripts are degraded during oocyte maturation, the proteins that participate in the SCMC persist until the early blastocyst stage. Genetic ablation of individual components provides evidence that this subcortical maternal complex is required for normal cleavage-stage mouse development. Among these, Filia and MATER proteins physically interact with one another and colocalize in the subcortex of eggs and early embryos. Both proteins are reversibly excluded from regions of cell-cell contact with subsequent restriction to the subcortex of "outer" cells and exclusion from "inner" cells, the precursors of the embryonic epiblast. Cell divisions orthogonal to the apical-basal axis of the polarized cell result in cell populations marked by the presence or absence of the FILIAMATER complex, even though the two component proteins persist in "inner" cells of the morula and ICM of blastocysts. Interestingly, the SCMC reforms in inner cells after release from cell-cell contacts and this may reflect the regulative nature of early mouse embryogenesis. These observations are consistent with a model in which the early embryo, although subject to regulative development, differentially accumulates a maternally expressed protein complex in topologically distinct blastomeres. The progeny of those containing the FILIA-MATER complex preferentially form the trophectoderm; those without the complex preferentially become the ICM of the blastocyst. However, the continued presence of the SCMC in "outer" cells of mutant embryos unable to establish trophectoderm, suggests that topology rather than cell lineage dictates the presence of the complex and that the SCMC does not appear to be sufficient to establish trophectoderm lineage.

The session ended with the talk by Wendy Dean, Laboratory of Developmental Genetics and Imprinting, The Babraham Institute, Cambridge (UK). She begun to report that in mammals, paternal and maternal pronuclei undergo profound chromatin reorganization upon fertilization. These observations together with others, lead Dean and collaborators to ask about another epigenetic facet, i.e. chromatin architecture, during these early events, first at the one cell stage and then during the preimplantation period up to the time of lineage determination. Remarkably, they found that the major and minor satellites of the centromere first organized around the nucleolar precursor bodies, a region know to harbor heterochromatin regions of the genome, independent of the chromatin difference between male and female chromatin. Thereafter, they observed that prochromocentric organization was distributed in an apparently uniform manner across the genome. By the 8-cell stage, these chromocentric structures began to polarize such that by the blastocyst stage, distinct patterns characteristic of the ICM and trophectoderm (TE) were evident. These patterns could also be resolved by using ES cells to model the ICM and the TE cells, suggesting an underlying epigenetic aspect to the establishment of the two lineages. In support of an epigenetic role in lineage determination is the observed differential DNA methylation detected using an antibody to 5 methyl cytosine, where the ICM is hypermethylated compared to the TE.

All presentations and wide discussion accompanying them clearly showed that at the beginning of this new century, the biology of reproduction is alive and well. Several classical topics within the field of the development and function of reproductive organs continue to intrigue and fascinate researchers, many of which find renewed strength from the overbearing entry of Stem Cell Biology into the field. Upon leaving the Meeting, most researchers agreed to meet again in Edinburgh for the $\mathrm{XV}$ Workshop of the series.

\begin{abstract}
Massimo De Felici ${ }^{1}$ and Rita Canipari²
${ }^{1}$ Department of Public Health and Cell Biology, University of Rome Tor Vergata and ${ }^{2}$ Department of Histology and Medical Embryology, University of Rome La Sapienza, Rome, Italy
\end{abstract}

KEY WORDS: reproduction, oocyte, sperm, gonad, ovary, testis, primordial germ cell 


\section{Further Related Reading, published previously in the Int. J. Dev. Biol.}

See our recent Special Issue Fertilization, in honor of David L. Garbers and edited by Paul M. Wassarman and Victor D. Vacquier at:

http://www.ijdb.ehu.es/web/contents.php?vol=52\&issue=5-6

Imprinting of mammalian male gametes is gene specific and does not occur at a single stage of differentiation

María D. Boyano, Noelia Andollo, María M. Zalduendo and Juan Aréchaga

Int. J. Dev. Biol. (2008) 52: 1105-1111

Pluripotency and differentiation in embryos and stem cells - Pavia, 17-18

January 2008

James A. Adjaye, Anne G. Byskov, Jose B. Cibelli, Ruggero De Maria, Stephen Minger,

Maurilio Sampaolesi, Giuseppe Testa, Catherine Verfaillie, Magdalena ZernickaGoetz, Hans Schöler, Michele Boiani, Nicola Crosetto and Carlo A. Redi Int. J. Dev. Biol. (2008) 52: 801-809

Puzzles of mammalian fertilization-and beyond

J.. Michael Bedford

Int. I. Dev. Biol. (2008) 52: 415-426

Interleukin-2 induces the proliferation of mouse primordial germ cells in vitro Cristina Eguizabal, Maria D. Boyano, Alejandro Díez-Torre, Ricardo Andrade, Noelia Andollo, Massimo De Felici and Juan Aréchaga

Int. J. Dev. Biol. (2007) 51: 731-738

Where do we stand now? - mouse early embryo patterning meeting in Freiburg, Germany (2005)

Takashi Hiiragi, Vernadeth B. Alarcon, Toshihiko Fujimori, Sophie Louvet-Vallée, Marek Maleszewski, Yusuke Marikawa, Bernard Maro and Davor Solter Int. J. Dev. Biol. (2006) 50: 581-588

\section{Primordial germ cell migration}

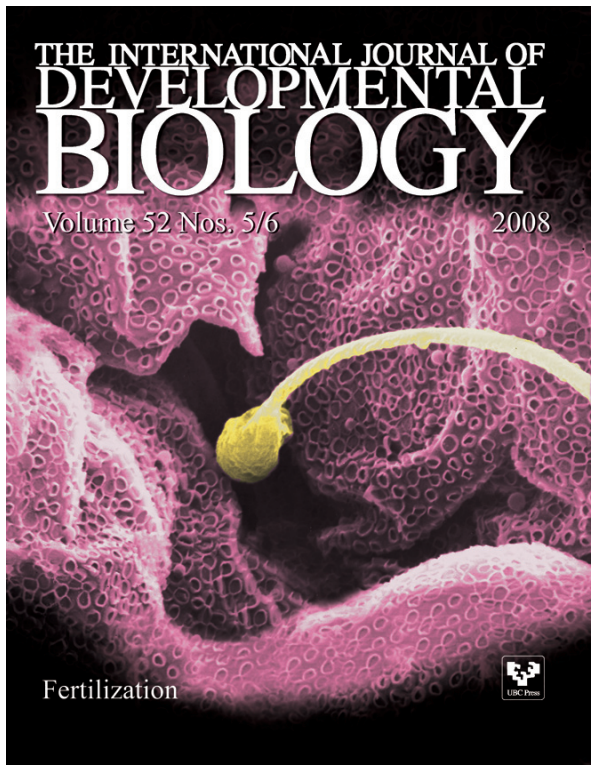

\section{For all the latest on Epigenetics research, see our latest Special Issue edited by Saadi Khochbin and Stefan Nonchev}

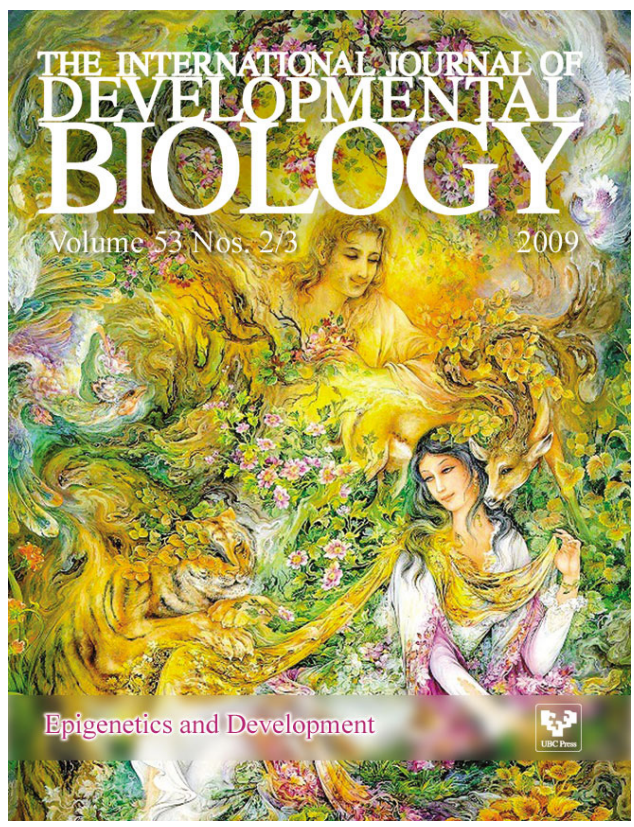

